\title{
The Population of AGNs in Nearby Galaxies
}

\author{
Luis C. Ho \\ The Observatories of the Carnegie Institution of Washington, 813 Santa \\ Barbara St., Pasadena, CA 91101, U.S.A.
}

\begin{abstract}
This contribution reviews the properties of nuclear activity in nearby galaxies, with emphasis on results obtained from current optical surveys and multiwavelength follow-up observations thereof.
\end{abstract}

\section{Introduction}

In a meeting which celebrates different techniques of surveying AGNs, it is appropriate to remind ourselves of the properties of the AGN population in nearby galaxies. Nearby AGNs are important for at least two reasons. First, they inform us of the faint end of the local $(z \approx 0)$ AGN luminosity function, a fundamental constraint on a variety of statistical considerations of the AGN population. Second, as the evolutionary endpoints of quasars, they present an opportunity to study black hole accretion in a unique regime of parameter space. This paper limits itself to three topics. Section 2 summarizes some general statistics resulting from optical searches for nearby AGNs. The interpretation of these results largely hinges on our understanding of the physical origin of low-ionization nuclear emission-line regions (LINERs; Heckman 1980), which is the subject of Section 3. Finally, Section 4 draws some inferences on the demographics of massive black holes.

\section{Statistics of Nearby AGNs from the Palomar Survey}

Most AGN surveys rely on selection criteria that isolate some previously known characteristics of these objects. Common strategies to find quasars, for example, employ color cuts to highlight the UV excess typically present in AGN spectra, or objective prism plates to identify objects with strong emission lines. Other wavelength-specific techniques to find candidate AGNs include combing areas of the sky in the radio or X-rays. Infrared-based methods use temperature selection to cull sources "warmer" than might be expected for star-forming galaxies. While all of these techniques have been successful, each introduces biases specific to the wavelength. And all require follow-up optical spectroscopy to confirm the AGN identification, to classify its type, and to determine its redshift.

A more direct, less biased approach is to spectroscopically survey every object within a particular region of the sky to a given optical magnitude limit. This, of course, is an expensive route, and in practice one is confined to go deep over only a small solid angle or stay relatively shallow over a wider area. In addition, when covering a wide area, a morphological cut has to be made so that one does not waste a lot of time observing foreground stars. 


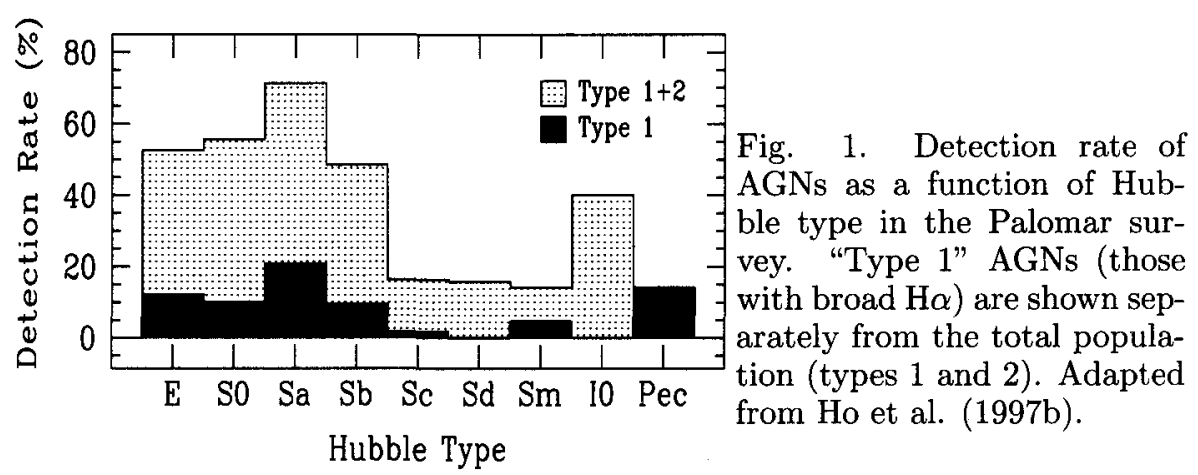

The results presented in the rest of this paper stem from the the Palomar optical spectroscopic survey of nearby galaxies (Filippenko \& Sargent 1985; Ho, Filippenko \& Sargent 1995). In brief, the Palomar 200-inch telescope was employed to take moderate-dispersion, high-quality spectra of 486 bright $\left(B_{T} \leq\right.$ $12.5 \mathrm{mag})$, northern $\left(\delta>0^{\circ}\right)$ galaxies, with the primary aim of conducting an accurate census of the AGN population in the nearby $(z \approx 0)$ universe. The Palomar survey, the most sensitive of its kind (see Ho 1996 for a comparison with previous optical studies), produced a comprehensive, homogeneous catalog of spectral classifications of nearby galaxies (Ho et al. 1997a, 1997b, 1997c). The main results of the survey are the following. (1) AGNs are very common in nearby galaxies (Fig. 1). At least $40 \%$ of all galaxies brighter than $B_{T}=12.5$ mag emit AGN-like spectra. The emission-line nuclei are classified as Seyferts, LINERs, or transition objects (LINER/H II), and most have very low luminosities compared to traditionally studied AGNs. The luminosities of the $\mathrm{H} \alpha$ emission line range from $10^{37}$ to $10^{41} \mathrm{erg} \mathrm{s}^{-1}$, with a median value of $\sim 10^{39} \mathrm{erg}$ $\mathrm{s}^{-1}$. (2) The detectability of AGNs depends strongly on the morphological type of the galaxy, being most common in early-type systems (E-Sbc). The detection rate of AGNs reaches $50 \%-75 \%$ in ellipticals, lenticulars, and bulge-dominated spirals but drops to $\$ 20 \%$ in galaxies classified as Sc or later. (3) LINERs make up the bulk (2/3) of the AGN population and a sizable fraction (1/3) of all galaxies. (4) A significant number of objects show a faint, broad (FWHM $\approx$ $1000-4000 \mathrm{~km} \mathrm{~s}^{-1}$ ) $\mathrm{H} \alpha$ emission line that qualitatively resembles emission arising from the conventional broad-line region of "classical" Seyfert 1 nuclei and quasars.

\section{The Nature of LINERs}

If LINERs ${ }^{1}$ are powered by a nonstellar central source, then they clearly would be the most abundant type of AGNs in nearby galaxies. However, ever since their discovery, the physical origin of LINERs has been controversial. The AGN

\footnotetext{
${ }^{1}$ Note that this paper is concerned only with compact, nuclear LINERs $(r \lesssim 200 \mathrm{pc})$, which are most relevant to the AGN issue. LINER-like spectra are often also observed in extended nebulae such as those associated with cooling flows, nuclear outflows, and circumnuclear disks.
} 


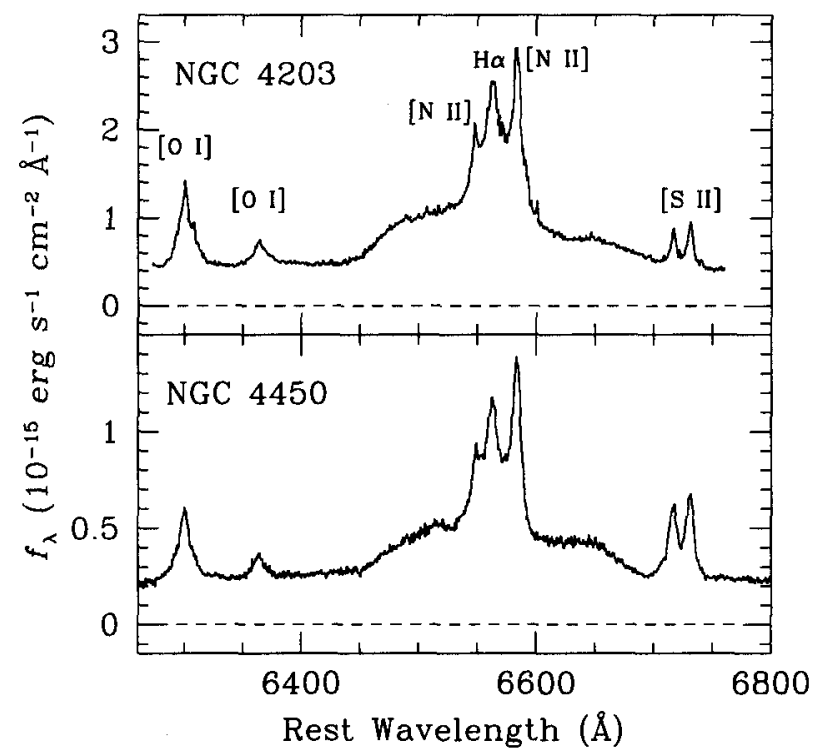

Fig. 2. Double-peaked broad $\mathrm{H} \alpha$ lines detected in STIS spectra of NGC 4203 (Shields et al. 2000) and NGC 4450 (Ho et al. 2000).

interpretation for LINERs is only one of several possible explanations, as recently reviewed by Barth (2001). The most uncertain subset are the type 2 LINERs and transition objects, whose energetics may arise, at least in part, from stellar processes.

Ho (1999a) used several lines of evidence to argue that a significant fraction of LINERs are genuine AGNs. Some of these are repeated below, along with updated information when available.

(1) The host galaxies of LINERs are similar to those of Seyfert nuclei. Both classes reside in bulge-dominated hosts (E-Sbc), quite distinctive from starforming H II nuclei, which are found mainly in Hubble types Sc and later. To the extent that bulges invariably contain massive black holes (e.g., Magorrian et al. 1998; Kormendy \& Gebhardt 2001; R. Green, these proceedings), this is consistent with the idea that LINERs are associated with accretion processes.

(2) Some of the best candidates for massive black holes are in LINER galaxies. Well-known examples include M81, M84, M87, and the Sombrero galaxy. The rapidly growing list of objects with kinematically determined black hole masses (see, e.g., Kormendy \& Gebhardt 2001; Ho 2001b) continues to support this.

(3) LINERs contain broad-line regions. The Palomar survey discovered that 15\%-25\% of the LINER population are "type 1" LINERs - LINERs with a directly visible broad component of $\mathrm{H} \alpha$ emission (Ho et al. 1997c). The broad-line component, however, is generally rather weak; the measurement is extremely challenging, requiring careful subtraction of the underlying starlight and deblending of complex line profiles. The robustness of the broad $\mathrm{H} \alpha$ detections, therefore, remained to be evaluated by independent observations. Rix et al. (2002) recently used STIS on HST to obtain nuclear spectra of a statistically complete subsample of emission-line nuclei selected from the original Palomar survey. The small aperture (0!'2) used in these observations excludes 

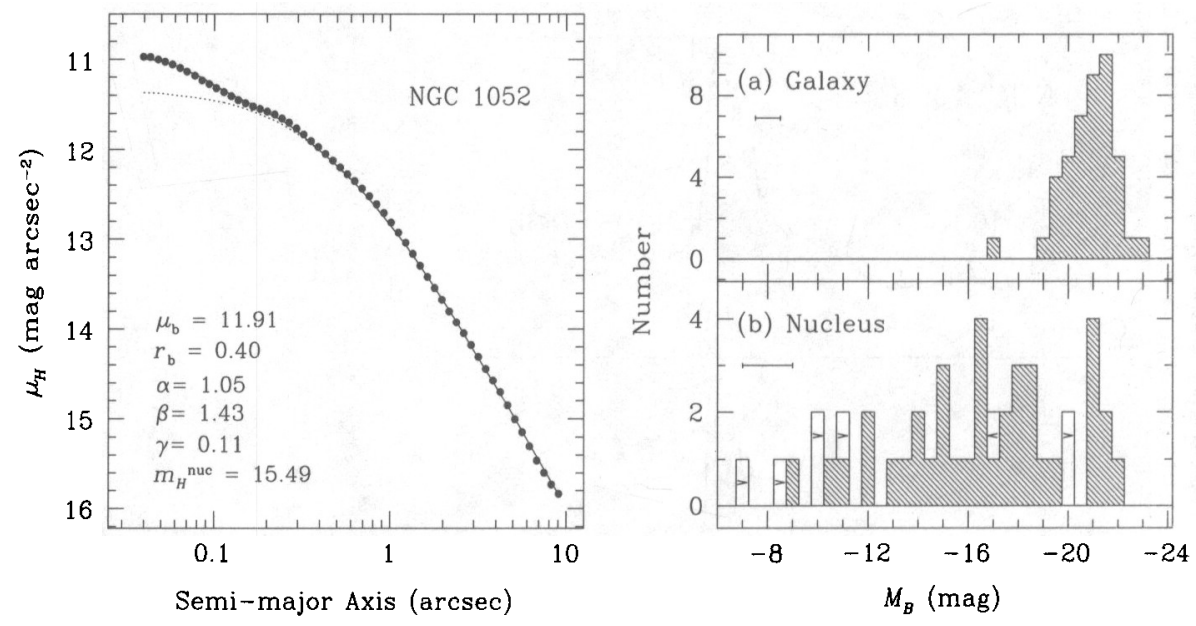

Fig. 3. (Left) NICMOS/HST $H$-band central surface brightness profile of NGC 1052. The central point source is extracted after modeling the underlying bulge light. Adapted from Ravindranath et al. (2001). (Right) Optical (B) absolute magnitudes for Seyfert 1 galaxies. The top panel plots the integrated emission from the galaxy, and the bottom panel shows the light from the nucleus alone, as determined from HST photometry. Adapted from Ho \& Peng (2001).

most of the contaminating bulge light and enables a much more straightforward assessment of the presence of broad lines. These data, and other related observations using $H S T$, have shown that the statistics of broad $\mathrm{H} \alpha$ emission derived from the Palomar survey are essentially robust (Rix et al. 2002).

However, the HST spectra revealed that in some objects (Fig. 2) the broad line does show a qualitative difference in the form of a double-peaked, asymmetric profile, highly reminiscent of similar structures found in some broad-line radio galaxies that are commonly interpreted as signatures of a relativistic accretion disk (e.g., Chen, Halpern, \& Filippenko 1989). Double-peaked broad lines are supposed to be quite rare; according to Eracleous \& Halpern (1994), they are seen in only $10 \%$ of radio-loud AGNs ( $\sim 1 \%$ of all bright AGNs). Given the relatively high detection frequency of these objects in serendipitous HST observations (M81: Bower et al. 1996; NGC 4203: Shields et al. 2000; NGC 4450: Ho et al. 2000; NGC 4579: Barth et al. 2001), we suspect that this phenomenon is much more common than previously thought. Indeed, Ho et al. (2000) discuss how double-peaked broad lines might arise naturally in nearby nuclei with low-accreting black holes.

(4) Compact nuclei are common in LINERs. But they are not easy to measure. Emission from the host galaxy invariably swamps the nuclear component. The contrast problem is not as severe at some wavelengths compared to others (e.g., UV), but generally it never goes away. In order to reliably quantify the nuclear fluxes from these sources, one needs observations not only with good sensitivity but also high angular resolution. The second point is crucial: generally angular resolutions of $\varsigma^{\prime \prime}$ are necessary to unambiguously disentable a tiny central core 
embedded in a bright bulge. The requisite data, although in principle not that challenging to obtain with modern facilities, have been subject to systematic analysis only quite recently.

Figure 3 illustrates the practical challenge. The left panel shows the central surface brightness profile of the well-studied LINER NGC 1052 measured in the $H$ band with NICMOS on HST (Ravindranath et al. 2001). The central point source is faint, but quite well defined at $r \leqslant 0^{\prime \prime} 1$. It almost certainly would have escaped notice in typical ground-based images. Even with $H S T$ resolution, however, note that it is not trivial to extract the point-source magnitude unambiguously in the presence of a cuspy bulge profile. The right panel in the figure generalizes this problem to a well-defined sample of nearby Seyfert 1 galaxies recently studied by Ho \& Peng (2001). It shows that although the contrast problem is most acute in LINERs, it is nonetheless quite important as well in Seyfert galaxies. The top panel, which plots the integrated optical $(B)$ absolute magnitudes of the entire galaxy, gives the familiar result that Seyfert galaxies typically have $M_{B} \approx-21 \mathrm{mag}$, roughly corresponding to $L^{*}$ (e.g., Ho et al. 1997b). On the other hand, the true magnitude of the Seyfert nucleus - the quantity most pertinent to the AGN phenomenon and most analogous to observations of quasars - ranges from $M_{B} \approx-21$ to -9 , a factor up to $10^{5}$ fainter than the integrated luminosity. Clearly, any quantitative discussion of the physical properties of low-luminosity AGNs, be they LINERs or Seyferts, would be meaningless if this were not taken into account.

The incidence of compact nuclei in LINER galaxies depends on wavelength, but in general the detection rate is quite high, on the order of $50 \%$ or greater. The most robust statistics come from radio and $\mathrm{X}$-ray observations, since these bands are least affected by dust obscuration.

Several recent studies have exploited the high angular resolution of the VLA to search for compact radio cores in the Palomar galaxies (Van Dyk \& Ho 1998; Nagar et al. 2000; Filho, Barthel, \& Ho 2000; Ho \& Ulvestad 2001). The VLA can deliver fairly sensitive ( $\mathrm{rms} \sim 50-100 \mu \mathrm{Jy}$ ) radio continuum images efficiently in "snapshot" mode. In general the observations have been done at 6 , 3.6 , or $2 \mathrm{~cm}$, with resolutions of $\sim 1^{\prime \prime}$. The main results are: (a) compact cores are quite common, being present in $\sim 50 \%-80 \%$ of the objects; (b) they are weak, typically $P_{6 \mathrm{~cm}} \approx 10^{18}-10^{21} \mathrm{~W} \mathrm{~Hz}^{-1}$; (c) a sizable fraction of them have flat or even inverted spectra; and (d) they have relatively simple structures, usually well described by a single unresolved core. Many sources remain unresolved when examined at milli-arcsecond resolution using VLBI techniques (e.g., Wrobel, Fassnacht, \& Ho 2001; Filho, Barthel, \& Ho 2001; Ulvestad \& Ho 2002, in preparation), although some show jetlike linear extensions (Falcke et al. 2000).

The Palomar galaxies have also been the subject of intense scrutiny in the $\mathrm{X}$-rays, both in the soft-energy band using ROSAT (Komossa, Böhringer, \& Huchra 1999; Roberts \& Warwick 2000; Halderson et al. 2001) and in the hardenergy band using $A S C A$ (Terashima, Ho, \& Ptak 2000; Terashima et al. 2001). The coarse angular resolution of these satellites, however, severely limits one's ability to reliably measure the weak signal from the nucleus. This is the domain of Chandra. A large, well-defined subsample of the Palomar galaxies is currently being imaged with ACIS. The preliminary findings, reported by Ho et al. (2001), suggest that compact $\mathrm{X}$-ray sources astrometrically coincident with the radio 

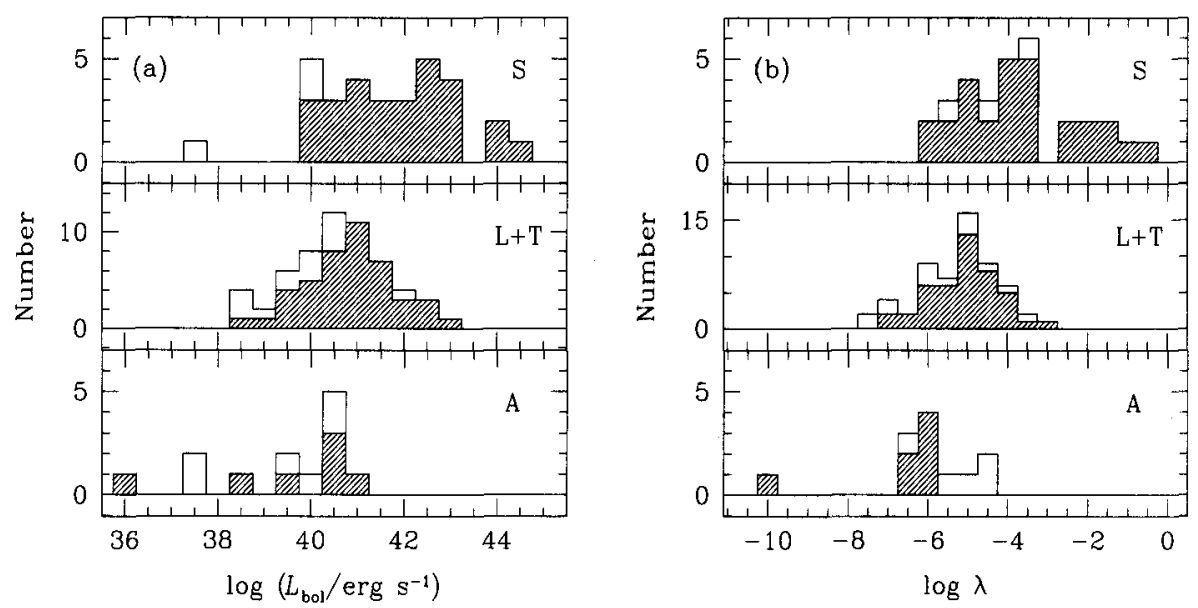

Fig. 4. Distribution of ( $a$ ) nuclear bolometric luminosities and (b) Eddington ratios $\lambda \equiv L_{\text {bol }} / L_{\text {Edd }}$ for Seyferts (S), LINERs and transition objects (L+T), and absorption-line nuclei (A). Open histograms denote upper limits. Adapted from Ho (2002, in preparation).

cores are again commonplace. They are detected universally in type 1 LINERs and Seyferts, relatively often in type 2 LINERs and Seyferts, but very rarely in transition nuclei. These statistics need to be verified with the full sample, which is forthcoming (Ho et al. 2002, in preparation).

(5) LINERs are highly sub-Eddington systems. Recent advances in black hole mass determinations in nearby galaxies gives us an opportunity to compare the nuclear luminosities described above with the corresponding Eddington luminosities. Ho (2002, in preparation) estimated nuclear bolometric luminosities for 100 Palomar galaxies based on X-ray measurements. As shown in Figure $4 a$, the majority of the objects have $L_{\text {bol }} \lesssim 10^{42} \mathrm{erg} \mathrm{s}^{-1}$, with the interesting trend that Seyferts are systematically more luminous than LINERs (including transition objects), which in turn are brighter than absorption-line nuclei (objects with no nuclear optical emission lines). More revealing still is the trend with $\lambda \equiv L_{\text {bol }} / L_{\text {Edd }}$ (Fig. $4 b$ ). The distributions of $\lambda$ systematically shift to lower values following the sequence $\mathrm{S} \rightarrow \mathrm{L}+\mathrm{T} \rightarrow \mathrm{A}$. Although LINERs and Seyferts broadly overlap, note that all LINERs are characterized by $\lambda \lesssim 10^{-3}$.

(6) The spectral energy distributions (SEDs) are peculiar. Specifically, the SEDs generically lack the optical-UV "big blue bump" (Ho 1999b; Ho et al. 2000), a near-universal feature of unobscured high-luminosity AGNs usually attributed to thermal emission from an optically thick, geometrically thin accretion disk. Another attribute of the SEDs of low-luminosity AGNs, especially of LINERs, is that they are typically "radio loud," defined here by the convention that the radio-to-optical luminosity ratio exceed some fiducial value, say $R>10$, as normally adopted in quasar studies. In fact, Ho (2001b) finds that among the $\sim 40$ nearby galaxies with kinematically determined black hole masses, essentially 


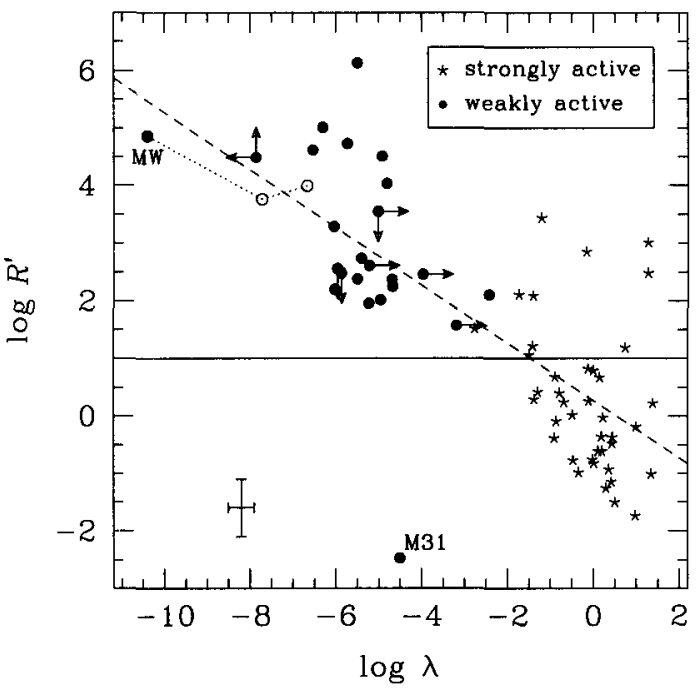

Fig. 5. Distribution of the nuclear radio-tooptical luminosity ratio $R^{\prime}$ vs. $\lambda \equiv L_{\text {bol }} / L_{\text {Edd }}$. The solid line marks the formal division between radioloud and radio-quiet objects, $R^{\prime}=10$. The dashed line is the best-fitting linear regression line. Adapted from Ho (2001b).

all are radio loud (Fig. 5). Since many of these objects are spiral galaxies, this result challenges the conventional wisdom that radio loudness is an attribute unique to giant ellipticals (see Ho \& Peng 2001 for a related discussion in the context of Seyfert galaxies). Moreover, Figure 5 shows that the degree of radio loudness evidently increases systematically with decreasing accretion rate, here parameterized by $\lambda$.

(7) LINERs have nonstandard central engines. As further elaborated in Ho et al. (2000) and Ho (2001a), LINERs share several characteristics expected of massive black holes fed by an advection-dominated accretion flow (see reviews by Narayan, Mahadevan, \& Quataert 1998; Quataert 2001) instead of a canonical thin accretion disk. The most pertinent of these are (a) the low Eddington ratios and the inferred low mass accretion rates, (b) the absence of the big blue bump in the SEDs, (c) the characteristic radio loudness and its dependence on $L_{\text {bol }} / L_{\mathrm{Edd}}$, (d) the prevalance of double-peaked broad emission lines and the disk structure they imply, and (e) the low-ionization state of the line-emitting gas, a possible consequence of the particular form of the SEDs.

(8) The nature of type 2 LINERs and transition objects is uncertain. An important unsolved problem is what fraction of the narrow-lined sources should be considered genuine AGNs. To be sure, AGN-like LINER 2 s do exist. Barth, Filippenko, \& Moran (1999a) discovered that the LINER 2 nucleus of NGC 1052 shows prominent broad-line emission when viewed in scattered light. The unification scheme, popular for Seyfert galaxies, evidently applies to at least some LINERs. If one assumes that all LINERs strictly adhere to the simplest form of the unified model (that all type 2 sources are simply obscured type 1 sources), and that the ratio of LINER $2 \mathrm{~s}$ to LINER $1 \mathrm{~s}$ is the same as that of Seyfert 2s to Seyfert $1 \mathrm{~s}$ in the Palomar survey, then the true AGN fraction among all LINERs is estimated to be between $45 \%$ and $65 \%$, depending on whether transitions objects are excluded or included, respectively (Ho 1996, 1999a). This estimate may be overly optimistic for at least two reasons. First, the spectropolarimetric observations of these very faint sources are demanding and still quite limited 


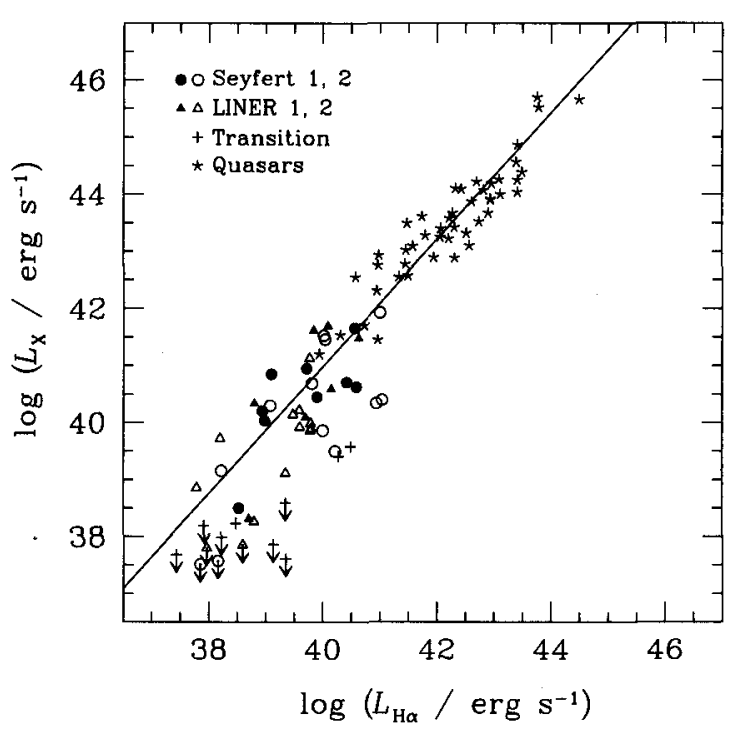

Fig. 6. Correlation between $2-10 \mathrm{keV}$ X-ray luminosity and $\mathrm{H} \alpha$ luminosity for AGNs of various types. Adapted from Ho et al. (2001).

(Barth et al. 1999a, 1999b), and so it may be unwise to generalize these results prematurely. And second, young, massive stars have been found to contribute significantly to the energy budget of some narrow-lined low-ionization objects (e.g., Maoz et al. 1998; Barth \& Shields 2000). The first caveat may be partly offset by the fact that some genuine low-luminosity AGNs (e.g., M84, M87, Sombrero) may intrinsically lack a broad-line region (Barth 2001).

The resolution of this problem requires access to data which are sensitive to a spectral region that is universally present in AGNs and is relatively unaffected by dust obscuration or modest photoelectric absorption. To be effective, the data must have high angular resolution; to survey large samples, necessary for statistical confidence, the data need to be gathered in an efficient mode. As described above (item 4), the current generation of radio and hard X-ray data both meet these requirements; we give greater weight to the latter because it carries a larger fraction of the AGN bolometric luminosity. Figure 6, taken from the preliminary results of the Chandra/ACIS survey of Ho et al. (2001), illustrates the potential of the X-ray data. Type 1 AGNs (LINERs, low-luminosity Seyferts, classical Seyferts, and quasars) obey a well-defined, linear correlation between hard X-ray $(2-10 \mathrm{keV})$ and $\mathrm{H} \alpha$ luminosity, down to $L_{\mathrm{X}}(2-10 \mathrm{keV}) \approx$ $3 \times 10^{38} \mathrm{erg} \mathrm{s}^{-1}$. The $L_{\mathrm{X}}-L_{\mathrm{H} \alpha}$ correlation strongly supports the hypothesis that all of these sources, which span $\sim 8$ orders of magnitude in luminosity, share the same physical origin.

Note that the majority of the type 2 sources (LINERs and Seyferts) $d o$ have compact X-ray cores. They follow the type 1 objects in Figure 6, albeit with somewhat greater scatter and offset toward lower $L_{\mathrm{X}} / L_{\mathrm{H} \alpha}$ by about a factor of 10. Interestingly, the X-ray hardness ratios of these sources suggest that absorption is not the culprit for their X-ray weakness. The transition objects, on the other hand, appear distinctly different as a class: most are undetected in X-rays, with upper limits as low as $L_{\mathrm{X}}(2-10 \mathrm{keV}) \approx 3 \times 10^{37} \mathrm{erg} \mathrm{s}^{-1}$. The incidence of compact radio cores in these objects (Filho et al. 2000) also appears to be lower than in LINERs or Seyferts. 


\section{Implications for Black Hole Demography}

To the extent that an AGN signature signifies accretion onto a massive black hole, a local AGN census gives us a lower limit on the fraction of nearby galaxies hosting massive black holes. If we accept that all LINERs, transition objects, and Seyferts are genuine AGNs, then the AGN statistics from the Palomar survey (Fig. 1) imply that black holes exist in $>40 \%$ of all galaxies with $B_{T} \leq 12.5$ mag. For bulge-dominated systems (E-Sbc), this fraction climbs to $>50 \%-75 \%$ - not inconsistent with the $100 \%$ claimed by direct kinematic studies. But how confident are we about the AGN-ness of nearby emission-line nuclei? The status of objects classified as Seyferts does not seem to be in dispute (for reasons that reflect historical bias more than concrete evidence). In any case, LINERs make up the majority of the population, and so deservedly receive closer scrutiny. The evidence presented in $\S 3$ shows that type 1 LINERs almost surely must be AGNs. The verdict for type 2 LINERs is much less clear-cut at the moment. While some sources have been shown to be powered by hot stars, others clearly have all the hallmark attributes of bona fide AGNs. Perhaps the most telling statistic is the incidence of compact radio and hard X-ray cores; although not yet well quantified, the preliminary results indicate that the detection rate is quite high in both bands $(z 50 \%)$. On the other hand, the "transition objects," postulated to be composite LINER/H II systems, may very well turn out to be unrelated to AGNs. Or if present, the AGN component must be significantly weaker than in "regular" LINERs. If we conservatively exclude all transition objects from the AGN pool, then the overall AGN fraction should be reduced by $13 \%$ (Ho et al. $1997 \mathrm{~b}$ ).

Acknowledgments. L.C.H. acknowledges financial support through NASA grants from the Space Telescope Science Institute (operated by AURA, Inc., under NASA contract NAS5-26555).

\section{References}

Barth, A. J. 2001, in Issues in Unification of AGNs, ed. R. Maiolino, A. Marconi, \& N. Nagar (San Francisco: ASP), in press

Barth, A. J., Filippenko, A. V., \& Moran, E. C. 1999a, ApJ, 515, L61

—. 1999b, ApJ, 525, 673

Barth, A. J., Ho, L. C., Filippenko, A. V., Rix, H.-W., \& Sargent, W. L. W. 2001, ApJ, 546, 205

Barth, A. J., \& Shields, J. C. 2000, PASP, 112, 753

Bower, G. A., Wilson, A. S., Heckman, T. M., \& Richstone, D. O. 1996, AJ, 111,1901

Chen, K., Halpern, J. P., \& Filippenko, A. V. 1989, ApJ, 339, 742

Eracleous, M., \& Halpern, J. P. 1994, ApJS, 90, 1

Falcke, H., Nagar, N. M., Wilson, A. S., \& Ulvestad, J. S. 2000, ApJ, 542, 197

Filho, M. E., Barthel, P. D., \& Ho, L. C. 2000, ApJS, 129, 93

-. 2001, MNRAS, submitted

Filippenko, A. V., \& Sargent, W. L. W. 1985, ApJS, 57, 503 
Halderson, E. L., Moran, E. C., Filippenko, A. V., \& Ho, L. C. 2001, AJ, 122, 637

Heckman, T. M. 1980, A\&A, 87, 152

Ho, L. C. 1996, in The Physics of LINERs in View of Recent Observations, ed. M. Eracleous et al. (San Francisco: ASP), 103

1999a, Adv. Space Res., 23 (5-6), 813

- $1999 \mathrm{~b}, \mathrm{ApJ}, 516,672$

2001a, in Issues in Unification of AGNs, ed. R. Maiolino, A. Marconi, \& N. Nagar (San Francisco: ASP), in press

-. 2001b, ApJ, in press

Ho, L. C., et al. 2001, ApJ, 549, L51

Ho, L. C., Filippenko, A. V., \& Sargent, W. L. W. 1995, ApJS, 98, 477

- 1997a, ApJS, 112, 315

$1997 \mathrm{~b}, \mathrm{ApJ}, 487,568$

Ho, L. C., Filippenko, A. V., Sargent, W. L. W., \& Peng, C. Y. 1997c, ApJS, 112,391

Ho, L. C., \& Peng, C. Y. 2001, ApJ, 555, 650

Ho, L. C., Rudnick, G., Rix, H.-W., Shields, J. C., McIntosh, D. H., Filippenko, A. V., Sargent, W. L. W., \& Eracleous, M. 2000, ApJ, 541, 120

Ho, L. C., \& Ulvestad, J. S. 2001, ApJS, 133, 77

Komossa, S., Böhringer, H., \& Huchra, J. P. 1999, A\&A, 349, 88

Kormendy, J., \& Gebhardt, K. 2001, in The 20th Texas Symposium on Relativistic Astrophysics, ed. H. Martel \& J. C. Wheeler (AIP), in press

Magorrian, J., et al. 1998, AJ, 115, 2285

Maoz, D., Koratkar, A. P., Shields, J. C., Ho, L. C., Filippenko, A. V., \& Sternberg, A. 1998, AJ, 116, 55

Nagar, N. M., Falcke, H., Wilson, A. S., \& Ho, L. C. 2000, ApJ, 542, 186

Narayan, R., Mahadevan, R., \& Quataert, E. 1998, in The Theory of Black Hole Accretion Discs, ed. M. A. Abramowicz, G. Björnsson, \& J. E. Pringle (Cambridge: Cambridge Univ. Press), 148

Quataert, E. 2001, in Probing the Physics of AGNs by Multiwavelength Monitoring, ed. B. M. Peterson et al. (San Francisco: ASP), 71

Ravindranath, S., Ho, L. C., Peng, C. Y., Filippenko, A. V., \& Sargent, W. L. W. $2001, \mathrm{AJ}, 122,653$

Rix, H.-W., et al. 2002, in preparation

Roberts, T. P., \& Warwick, R. S. 2000, MNRAS, 315, 98

Shields, J. C., Rix, H.-W., McIntosh, D. H., Ho, L. C., Rudnick, G., Filippenko, A. V., Sargent, W. L. W., \& Sarzi, M. 2000, ApJ, 534, L27

Terashima, Y., Ho, L. C., \& Ptak, A. F. 2000, ApJ, 539, 161

Terashima, Y., Iyomoto, N., Ho, L. C., \& Ptak, A. F. 2001, ApJS, in press

Van Dyk, S. D., \& Ho, L. C. 1998, in IAU Symp. 184, The Central Regions of the Galaxy and Galaxies, ed. Y. Sofue (Dordrecht: Kluwer), 489

Wrobel, J. M., Fassnacht, C. D., \& Ho, L. C. 2001, ApJ, 553, L23 